\section{Better informed in clinical practice - a brief overview of dental informatics}

\author{
P. A. Reynolds, ${ }^{1}$ J. Harper ${ }^{2}$ and S. Dunne ${ }^{3}$
}

VERIFIABLE CPD PAPER
IN BRIEF

- This paper develops the themes set out in the previous paper in this series and focuses on the development of dental informatics.

- In comparison with medical informatics, progress both in the United Kingdom and worldwide has been slow in comparison.

- The reasons for this and the current situation with regard to dental informatics are explored.

\begin{abstract}
Uptake of dental informatics has been hampered by technical and user issues. Innovative systems have been developed, but usability issues have affected many. Advances in technology and artificial intelligence are now producing clinically useful systems, although issues still remain with adapting computer interfaces to the dental practice working environment. A dental electronic health record has become a priority in many countries, including the UK. However, experience shows that any dental electronic health record (EHR) system cannot be subordinate to, or a subset of, a medical record. Such a future dental EHR is likely to incorporate integrated care pathways. Future best dental practice will increasingly depend on computer-based support tools, although disagreement remains about the effectiveness of current support tools. Over the longer term, future dental informatics tools will incorporate dynamic, online evidence-based medicine (EBM) tools, and promise more adaptive, patient-focused and efficient dental care with educational advantages in training.
\end{abstract}

\section{E-LEARNING IN DENTISTRY}

Section A: Teaching and technology

1. A description of the new technologies used in transforming dental education

2. Seeing is believing: dental education benefits from developments in videoconferencing

3. Webcasting: casting the web more widely

4. Top of the pops - CD-ROM and DVDs in dental education

Section B: Informatics: better informed by systems and services

5. Better informed: an overview of health informatics

6. Better informed in clinical practice a brief overview of dental informatics

7. Digital clinical records and practice administration in primary dental care

Section C: Impact of e-learning in

dental education

8. Remember the days in the old school yard: from lectures to online learning

9. An intricate web - designing and authoring a web-based course

10. The many faces of interaction

11. Supporting the learner and teacher online

12. Making a mark - taking assessment to technology

13. Continuing professional development and ICT: target practice

14. Assuring quality

Section D: A connected future

15. Nine years of DentEd: a global perspective

16. A vision of dental education in the third millenium

\section{INTRODUCTION}

A dental informatics program was one of the first applications ever to run on a computer in the days when there were less than twenty computers anywhere in the world and almost all were used either for military or physics research. Yet 'dental informatics' as a term does not appear in the literature until 1986, nearly twenty years after 'medical informatics' first appeared as a term.

Medical informatics itself had a delayed start. The first programs appeared in the 1950s. There was an astonishing burst of creativity in the 1960s: the first expert systems; the first clinical decision support systems; the first medical imaging programs; the first clinical information

\footnotetext{
"Professor of Dental Education, Centre of Flexible Learning in Dentistry, King's College London Dental Institute, Floor 3, Strand Bridge House, 138-142 The Strand, London, WC2 1HH; ${ }^{2}$ T Manager, The Dental Institute at Guy's, King's and St Thomas' NHS Hospitals, King's College Hospital, Denmark Hill, London, SE5 9RW; ${ }^{3}$ Professor of Primary Dental Care, The Dental Institute at Guy's, King's and St Thomas' NHS Hospitals, King's College Hospital, Denmark Hill, London, SE5 9RW ${ }^{*}$ Correspondence to: Professor P. A. Reynolds Email:p.a.reynolds@kcl.ac.uk
}

Refereed Paper

DOI: $10.1038 /$ bdj.2008.193

${ }^{\circledR}$ British Dental Journal 2008; 204: 313-317 systems. But then the momentum was lost. Breakthroughs at individual sites did not translate into general adoption of such technology across healthcare. The exception was the technically simpler and clinically less useful patient administration systems. It is only recently that medical informatics systems have begun to be adopted more generally.

Dental informatics had an even more delayed start. From the first dental informatics programs, over fifty years ago, to today, there has been much technical progress, but little widespread uptake. A recent conference in North America attended by most of that continent's leading researchers in the field, could describe dental informatics as 'emerging' and 'a small but growing discipline', nearly half a century after the first papers on dental informatics were published. In 2003, there were just two, NIDCR-NLM-funded, training programmes in dental informatics in North America, and neither predated $1997 .{ }^{1}$

In part, this delay has been a matter of policy and economics. Patient administration systems brought immediate benefits in terms of patient management and healthcare administration that clinical 
information systems did not. But medical and dental informatics has also faced major technical challenges. Despite the significant conceptual breakthroughs of the 1960s, all medical informatics, including dental, have faced major impediments in the shape of systems performance and integration issues.

\section{CHALLENGES IN DENTAL ICT}

Dental informatics is more than just the application of computing to dentistry. The earliest dental informatics pioneers described their approach as the application of information science to solving medical problems. ${ }^{2}$ The first definition of the term informatics stated that it was the study of 'the structure and general properties of scientific information' and the 'process of scientific communication'. ${ }^{3}$ More recent writers have described medical informatics as a cascade from analysis to effect. One proposes a four part structure: medical model formulation; system development; system installation; evaluation and modification. ${ }^{4}$ The challenge facing much dental informatics is the inherent difficulties at every step in this process.

Defining the medical model requires appropriate structured terms, medical vocabularies, taxonomies and ontologies. To work effectively in an informatics environment, these terms, concepts and structures must be both precise and highly standardised. Conventional medical vocabularies and coding systems are often ambiguous, and there is often wide divergence in practice between different specialties and different geographies. Medical coding systems such as Read Codes and SNOMED-CT are central to the success of large scale projects such as England's National Programme for IT, yet there is considerable debate as to their effectiveness.

The second stage in developing the software application is also a daunting and complex task. For example, translating all the information in a dental patient record into a format that is usable on a computer screen has proved in practice to be an extremely challenging operation. Some commentators ${ }^{5,6}$ have offered reasons why developing biomedical computer systems have proved so much more difficult than in other knowledge domains. Factors include the sheer complexity of much medical information, variability in the human body, and cognitive, ethical and emotional factors.

Attempts to exploit modern windows GUI environments to simplify and speed dental patient data input can have quite the reverse effect. Some windows-based dental systems can confuse the user with myriad sets of icons. One dental EPR system, described as 'a typical example of existing [dental] software', was, according to one reviewer, 'a challenge for users', with a toolset that was 'difficult to memorise'. The problem was the difficulty of matching an icon-based approach to data input with the complexity of dental information: 'The representation on the screen itself is packed with many diverse icons since a single tooth's possible findings consists of a minimum of four rows of information and, thus, lacks a quick overview and ease of use... many icons resemble one another in shape and colour and exceed in their total number the amount of pictorial representation of traditional paper charts'. The data input and management problem associated with the windows interface, however, is not confined to any one program: 'the points of concern mentioned here are valid for other dental software packages as well. ${ }^{7}$

New approaches to medical informatics that stress the importance of imaging, physiological data, unstructured biomedical data and computational analytics are being developed. These should result in medical and dental tools of far greater clinical use. However, a number of software and hardware challenges will have to be overcome before this new generation of tools becomes generally available.

The third level of Friedman's model is installation. For dental ICT, this is often a major hurdle. The low historical levels of uptake in dental ICT must be connected to the difficulty of translating research projects into clinically useful tools for dental practitioners. By the mid-1990s, university researchers had developed a range of expert systems for endodontics, oral radiology, oral pathology and removable prosthodontics. ${ }^{8}$ Just over five years later, none of these systems were still in use. ${ }^{1}$

\section{SPEECH RECOGNITION}

One significant reason for a low adoption rate of dental ICT tools is the unsuitability of the conventional computer interface for the typical dental practitioner. Computer keyboards and mice are a potential source of infection and cannot be used by the dentist while examining or treating the patient. They also tend to be slower input mechanisms than the traditional paper chart and pen. A dental assistant would typically take longer to enter data with a computer program and might be required anyway to assist the dentist. ${ }^{7}$

To make dental ICT more widespread in dental practice may well require the development of effective voice recognition technologies. It is increasingly being adopted in radiology, where a comparison of data from 5,072 reports generated by a commercial voice recognition system with 4,552 reports produced manually a year earlier show its potential to improve turn-around. ${ }^{9}$ In dentistry, voice recognition is still a work in progress. However, a development is underway to provide an integrated clinical dental record system accessible chairside by voice recognition or a wireless mouse. This system will be explained more fully in the next article in this series.

\section{DENTAL DECISION SUPPORT SYSTEMS}

One of the most promising categories of ICT tools for dentists is clinical decision support systems (CDSS). The outreach approach to dental training is gaining momentum, with students learning in practices away from the central hub. Having access to CDSS will ensure standards of engagement in this process.

CDSS are programs that are designed to support clinicians in making diagnoses and decisions about preventative and therapeutic care. In medicine, CDSS has aroused significant interest from doctors as they promise to support clinical practitioners in their core tasks. However there is considerable debate about just how effective the current generation of clinical decision support systems are.

There is increasing interest in CDSS becoming a mechanism for embodying an evidence-based medicine (EBM) 


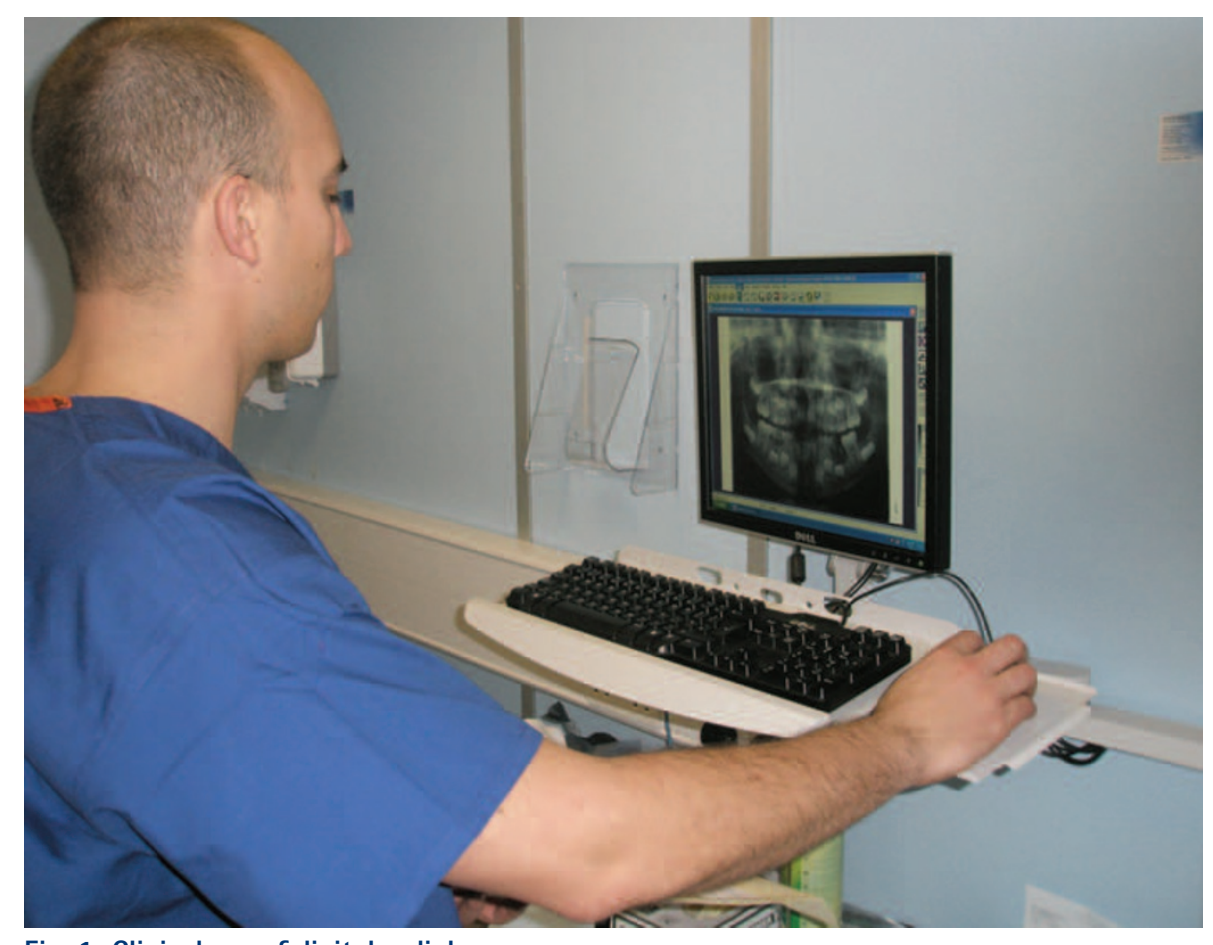

Fig. 1 Clinical use of digital radiology

approach to patient care. At present, most CDSS do not directly incorporate EBM, but future CDSS may incorporate literature trawling mechanisms that provide automatic EBM capabilities.

There has been development of dental decision support systems for over twenty years. ${ }^{7,10}$ In 1996, a comprehensive review of decision support systems in dentistry identified over thirty dental decision support systems. ${ }^{8}$ These were grouped into seven sub-areas of dentistry: dental emergencies and trauma, oro-facial pain, oral medicine, oral radiology, orthodontics, pulpal diagnosis and restorative dentistry. Amongst the systems identified by White were applications for the automated interpretation of radiographic lesions, dental radiographs, the landmark identification of cephalometric radiographs, treatment planning and removable partial denture design ${ }^{8}$ (Fig. 1).

Since White's review, other publications have described the development of dental decision support systems for oral surgery, caries management, pretherapy for patients with head and neck cancer and treatment planning.

The typical CDSS comprises four elements. The inference engine is the core of the system. It incorporates the logic system that allows inferences to be drawn. Knowledge bases hold the domain knowledge that the inference engine processes. Knowledge bases acquire their knowledge from external sources such as databases or may be built by a domain expert such as a specialist in oral cancer. Future CDSS are likely to acquire knowledge dynamically online by continual interrogation of the entire corpus of dental and medical literature, including 'submerged' data such as research trial raw data, unstructured data files and databases.

CDSS are generally seen as a powerful tool for supporting better decision making by the health professional. The UK health charity Isabel Healthcare has developed an award-winning CDSS in an explicit effort to improve the quality of primary care of acute patients, especially in the paediatric area. But many researchers are doubtful about the efficacy of older clinical decision support systems. In particular, algorithmic systems - the inferential technology in many older systems - may rely on narrow knowledge bases, and fail to comprehend complex clinical cases and clinical uncertainty. Algorithmic CDSS include systems for oral pathology. Other inference engine technologies also may suffer weaknesses.

Connecting for Health, the NHS agency charged with managing the National Programme for IT (NPfIT), ${ }^{10}$
England's $£ 6$ billion upgrade of the Health Service's IT infrastructure, has implemented a review of all CDSS currently employed by the NHS. It is concerned that some CDSS may be unreliable and provide inadequate warning of drug interactions.

The American Medical Informatics Association has concluded that traditional CDSS have had only a modest effect on improving prescribing while their impact on clinical outcomes is indeterminable but probably minor. It has called for a new class of 'evidenceadaptive' CDSS. These will be able to capture knowledge automatically from the corpus of clinical literature and research data, and apply such knowledge dynamically and flexibly to individual patients at point of care.

Developments in text mining and computational linguistics are likely to play a major role in the design of future dental decision support systems. Text mining technology is designed to uncover new knowledge from large numbers of unrelated text and unstructured data sources. It has already played a major role in certain areas of the biosciences both in uncovering the aetiology of rare diseases, an area pioneered at the University of Chicago, ${ }^{11}$ and in the emerging field of proteomics, where it is playing a key role in uncovering which proteins interact with which other proteins.

\section{DENTAL PATIENT RECORDS}

Moving from paper-based to electronic patient records has been a long term goal of most healthcare administrators. However, different stakeholder groups have different requirements from electronic patient records, which has tended to result in EPR systems designed for particular tasks.

Healthcare administrators seek EPR to facilitate the management and audit of healthcare provision. Policy makers believe that automation of patient records can produce significant cost savings. Policy makers associated with the English National Programme have asserted that trusts can fund part of the considerable local implementation costs of the programme from savings produced by automation. However, some practitioners believe that EPR could in 
practice drive up the overall cost of providing NHS healthcare. ${ }^{12}$

A major component of NPfIT is the introduction of a nationwide electronic health record (EHR) as well as a hierarchy of standardised EPRs in community, mental, primary and secondary care. As originally envisaged, this national programme does not include dentistry services. However in 2002, the NHS published Options for change. ${ }^{13}$ This called for the far closer integration of dental services into local primary care trusts, including the development of managed clinical dental networks to serve a similar function at PCT level as medical integrated care pathways.

Options for change has been followed by An information technology strategy for NHS dentistry in the 21st Century. ${ }^{14}$ Calling for dental EPRs to be developed within the evolving National Programme infrastructure, the report's authors caution that dental EPR should follow a different development track to the medical EPR, as its requirements would be significantly different from those the medical record. The report also underlined the importance of developing dental service integrated care pathways to extend evidence-based practice and clinical governance in dentistry.

However the systems develop, part of any student's core activity is learning to take a comprehensive history (Fig. 2). In maintaining records they will be expected to engage with both patients and the technology.

\section{TOWARDS THE FUTURE OF DENTAL ICT}

Three consecutive and continuing developments have been key in realising medical informatics as a practical set of tools for practitioners. The first, beginning from the late 1970s, has been a revolution in processor performance, largely the result of the development of RISC (reduced instruction set computer)-based technologies. True clinical applications require significant computing power. Many clinical and dental applications are today still at the very edges of what is possible computationally. Continuing increases in computing power will open up new possibilities in dental computing. This is especially true

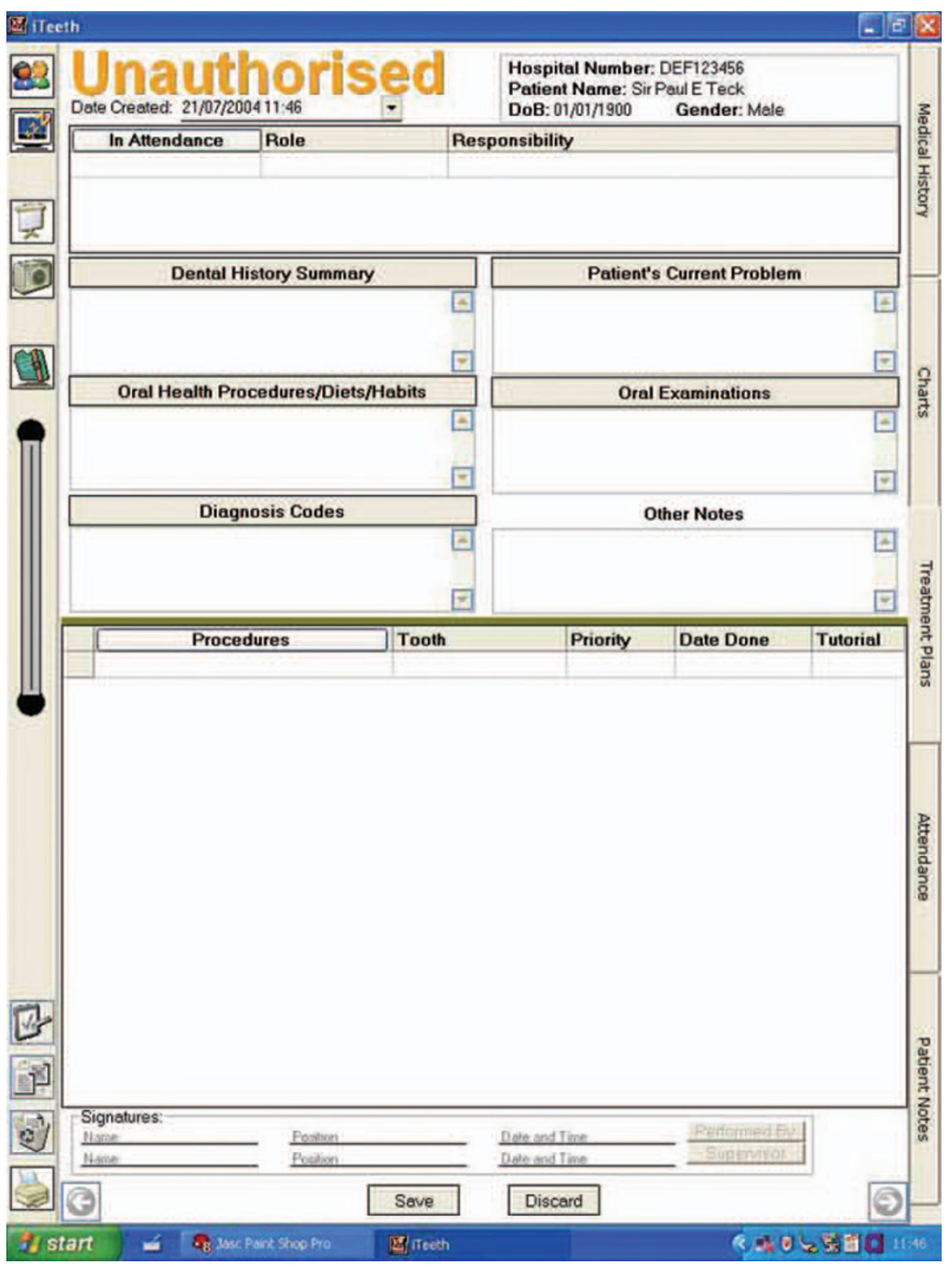

Fig. 2 Sample history taking form in an electronic patient record

of the emerging field of computational dentistry, which could have a significant effect on dental therapeutics, and the development of personalised dentistry, which could affect oral healthcare and cancer management. But increased computing power will also make possible the next generation of electronic dental patient records and dental decision support systems, which will incorporate analytics and dental imaging at their core.

The second development, starting in the late 1980s, has been the creation of standard, computer-coded medical vocabularies, semantic structures and medical data standards. Without these, building meaningful medical applications that can scale and integrate with other applications is impossible. The third development has been the emergence of biomedical open source methodologies and technologies. These are making possible enormous leaps forward in biomedical computation. The mapping of the human genome in such short time was made possible through open source.

This third wave, open source, is likely to play a key role in developing new dental technologies such as computational dentistry, which could bring new levels of accuracy and flexibility to dental diagnostics and therapeutics. Open source can also produce new solutions to old problems in dental informatics, such as cost and performance of hardware required to run advanced dental applications: open source tools such as Beowulf 
and Linux have been deployed to create supercomputer performance from commodity platforms such as games machines. Such configurations are not only cheap but with advanced real-time scalar imaging, they can provide sophisticated dental image processing to rival clusters of Unix servers costing hundreds of times more.

Another technology that has emerged from the open source movement is textomics, the marriage of text mining and biomedical data integration. Textomics was the core informatics technology underlying the human genome project. It has the potential to revolutionise both dental decision support systems and oral healthcare research. Textomics may be the key to the development of individual- ised medicine and dentistry, an approach being pioneered at Harvard Medical School and the Mayo Clinic and by a number of commercial organisations. Individualised medicine arises directly from the success of the human genome project; its premise is that genomics will shape patient care pathways, diagnostic and therapeutic interventions and lead to individualised treatments customdesigned for each patient.

1. Schleyer T K. Dental informatics: an emerging biomedical informatics discipline. Adv Dent Res 2003; 17: 4-8.

2. Ledley R S, Lusted $L B$. Reasoning foundations of medical diagnosis. Science 1959; 130: 9-21.

3. Collen M F. A history of medical informatics in the United States. Washington, DC: American Medical Informatics Association, 1995.

4. Friedman C P. Where's the science in medical informatics? J Am Med Inform Assoc 1995; 2: 65-67.

5. Maojo V, Martin F, Crespo J, Billhardt H. Theory, abstraction and design in medical informatics. Methods InfMed 2002; 41: 44-50.

6. Shahar Y. Medical informatics: between science and engineering, between academia and industry. Methods InfMed 2002; 41: 8-11.

7. Umar H. Capabilities of computerized clinical decision support systems: the implications for the practicing dental professional. J Contemp Dent Pract 2002; 1: 27-42.

8. White S C. Decision-support systems in dentistry. J Dent Educ 1996; 60: 47-63.

9. Ramaswamy M R, Chaljub G, Esch O, Fanning D D, vanSonnenberg E. Continuous speech recognition in MR imaging reporting: advantages, disadvantages, and impact. AJR Am J Roentgeno/ 2000; 174: 617-622.

10. NHS Connecting for Health website. http://www. connectingforhealth.nhs.uk (accessed 5 February 2008).

11. Swanson D R. Migraine and magnesium: eleven neglected connections. Perspect Biol Med 1988; 31: 526-557.

12. Wyatt J $C$, Keen J. The new NHS information technology. BMJ 2001; 322: 1378-1379.

13. Department of Health. NHS dentistry-options for change. London: Department of Health, 2002.

14. Department of Health. An information strategy. London: Department of Health, 2002. 